\author{
$\bullet \bullet \bullet \bullet$ \\ Der mentale Text. \\ Zu den \\ Entstehungsprozessen \\ von Kalevala und \\ Nibelungenlied \\ CHRISTIAN NIEDLING
}


Abstract Der Beitrag vergleicht die Diskussion der Epengenese des Nibelungenliedes im Spektrum der Theorien Karl Lachmanns und Andreas Heuslers und den so genannten Kalevala-Prozess. Beide Epen werden als semi-oral bzw. traditionsorientiert betrachtet. Den Ausgangspunkt der Untersuchung bilden die Prolegomena ad Homerum Friedrich August Wolfs von 1795 und die daraus entstandene Homerische Frage. Die Auseinandersetzung mit Wolf beeinflusste die Nibelungenforschung nachhaltig und stimulierte den so genannten Kalevala-Prozess in entscheidender Weise. Für die Entstehung des Kalevala sind auch die zeitgenössische Ossian-Debatte und Herders Einflüsse zu berücksichtigen.

Die Dokumentation der mündlichen finno-karelischen Sängertradition - der Dichtung hinter dem Kalevala - sowie des Epenprozesses des Kalevala ermöglicht die Betrachtung der Epenentstehung aus der traditionalistischen und der individualistischen Perspektive, $d$. h. der Überlieferungsprozesse, Variationsbildung und des Sänger-Individuums. Das Konzept des „mentalen Textes“ von Lauri Honko wird daran anknüpfend mit aktuellen Ansätzen der Nibelungenüberlieferung im semi-oralen bzw. traditionsorientierten Bereich verglichen.

Schlagworte Homerische Frage, Epentheorie, Semi-Oralität, Nibelungenlied, Kalevala

\section{Traditionsorientierte Epen}

Der finnische Folklorist Lauri Honko verbindet die Homerischen Epen, das Kalevala und das Nibelungenlied in einer Gattung (Honko 2000b):

„Die Homerischen Epen sollten als traditionsorientierte Epen klassifiziert werden, ebenso wie das Kalevala und die meisten der großen europäischen Epen wie Beowulf, das Rolandslied und das Nibelungenlied. Der Fakt, dass sie zur gleichen Kategorie gehören bedeutet, dass sie die gleiche oder eine vergleichbare Geschichte der Textualisierung haben. Die Einstellung ihrer Interpreten und Editoren zu ihrem traditionellen Material und Poetik unterschied sich 
in gewisser Weise. Gleichwohl teilen sie das Verlangen, die mündliche Dichtung, auf der sie basieren, zu erhalten und zu respektieren.“1

Epen, also umfangreiche narrative Dichtungen in einem gehobenen Stil über Heldentaten mit Bedeutung für die kulturelle Identität einer Gruppe (vgl. Honko 1995, S. 26), sind demnach traditionsorientiert, wenn sie auf mündlicher, identitätsstiftender Überlieferung basieren. Die tradierten Texte sind damit „Manifestationen einer überindividuellen Gedächtniskultur, die die Erzähler ebenso wie die Hörer zugleich bindet und orientiert.” (Heinzle 2005, S. 158) Die variantenreiche Sagentradition etwa des Nibelungenkomplexes ist als Teil des kollektiven Gedächtnisses anzusehen, über das sowohl Sänger wie Publikum verfügen (Schmid 2018, S. 16):

„Wie für mündliches so ist ebenso für schriftliches Wiedererzählen eines bekannten Stoffes nicht nur seitens des Produzenten, sondern auch seitens des Rezipienten Sagenkenntnis vorauszusetzen. Perspektivierungen des Stoffs, Um- und Neuformulierungen, vermeintliche oder tatsächliche Leerstellen des Texts - nicht nur aus neuzeitlicher Perspektive - werden vermutlich zur Kenntnis genommen, überbrückt, kontrastiert, vervollständigt usw. Jede (Neu-)Gestaltung muss sich mit dieser Tradition auseinandersetzen und findet eine eigene ,Antwort' auf Vorgaben und Erwartungen in der je spezifischen historischen Konstellation.”

Das von Honko eingangs erwähnte respektvolle Erhalten der Tradition bedeutet auch, dass Varianten berücksichtigt werden. Joachim Heinzle spricht in diesem Kontext von der „Integration des Divergenten“(Heinzle 2014, S. 130) und sowohl Heinzle als auch Honko verweisen darauf, dass wir uns die mündliche Erzähltradition gar nicht „vielgestaltig und widersprüchlich genug vorstellen können“ (Heinzle 2014, S. 157; vgl. Honko et al. 1993) - viel variantenreicher, als es die überlieferten Dokumente ohnehin erahnen lassen.

1 Soweit nicht anders vermerkt, stammen die Übersetzungen von CN. Im Beitrag werden der leichteren Lesbarkeit halber generische Maskulina für alle Geschlechter verwendet. 
Zwischen dem historischen Geschehen der im Nibelungenlied geschilderten Ereignisse und den ersten schriftlichen Zeugnissen ab 1200 haben wir es mit einem langen Zeitraum mündlicher Überlieferung und Variantenbildung zu tun: Der historisch fassbare Kern des Nibelungenliedes, der Untergang der Burgunden im zweiten Teil des Epos, geht auf Ereignisse des 5. Jahrhunderts zurück: Die Burgunder erlitten um 436 eine vernichtende Niederlage gegen den römischen Feldherrn Aëtius und mit ihm verbündete hunnische Hilfstruppen. Nach der Vernichtung großer Teile des Stammes und des Königshauses wurden die verbleibenden Burgunder 461 in die Region Lugdunum um das heutige Lyon umgesiedelt. Dort ließ König Gundobad (gestorben 516) das Stammesgesetz niederschreiben, die Lex Burgundionum. Darin werden als dessen Vorgänger auch Gibica (mhd. Gibiche, nord. Gjuki; im Nibelungenlied Dancrat bezeichnet), Gundaharius (mhd. Gunther, nord. Gunnar), Gislahari (mhd. Giselher, keine nord. Entsprechung) sowie Gundomaris (mhd. Gernot, nord. Guttorm) genannt.

Attila (geb. 406; mhd. Etzel, nord. Atli) und dessen Bruder Bleda (mhd. Blödelin), waren anders als im Nibelungenlied nicht am Untergang der Burgunden beteiligt. Offenbar liegt hier eine Vermischung mit der Schlacht auf den Katalaunischen Feldern des Jahres 451 vor, in der auch Attila eine Niederlage gegen Aëtius erlitt. Der Tod ereilte den Hunnenherrscher im Jahre 453 im Bettlager mit der Germanin Hildico. Hinter Dietrich von Bern schließlich steht Ostgotenkönig Theoderich der Große, der wiederum kein Zeitgenosse Attilas war: Er wurde um 454 geboren und starb 526. Auch diese aus heutiger Sicht ahistorische Darstellung ist ein Merkmal der Vorzeitkunde. Bald nach diesen Ereignissen entstand die Nibelungensage bei den Burgunden und Franken. Sie wurde über Jahrhunderte mündlich tradiert und verbreitete sich spätestens ab dem 10. Jahrhundert in Nordeuropa (vgl. Heinzle 2014, S. 128), wo sie sich ganz offensichtlich sehr großer Beliebtheit erfreute und mit einheimischen Stoffen vermischte. Die erste Verschriftlichung erfolgte im deutschsprachigen Raum, aber im Norden haben sich ältere Stufen erhalten, die später gesammelt und niedergeschrieben wurden: in den Edda-Liedern, der Thidrekssaga und der Völsungasaga.

Dem Sammelinteresse der Eddas sind auch Hinweise auf umlaufende Parallelversionen von Liedern zu verdanken. Zwei Beispiele sollen dies 
verdeutlichen: Im Brot af Sigurðakviðu der Lieder-Edda werden der Bett- und der Wald-Tod Sigurds als Varianten erwähnt:

„Frá dauða Sigurðar. Hér er sagt í Pessi kviðo frá dauða Sigurðar, ok víkr hér svá til, sem Peir dræpi hann úti. En umir segia svá, at Peir dræpi hann inni í rekkio sinni sofanda. En býðverskir menn segia svá, at beir dræpi hann úti í skógi. Ok svá segir í Guðrúnarkviðo inni forno, at Sigurðr ok Giúka synir hefði til pings riðit, Pá er hann var drepinn. En Pat segia allir einnig, at Peir sviko hann í tryggð ok vógo at hánom liggianda ok óbúnom.” (Neckel 1914, S. 196)

(„Vom Tode Sigurds. Hier in diesem Lied wird vom Tode Sigurds gesprochen. Und es läuft darin so ab, als hätten sie ihn draußen getötet. Einige jedoch sagen, dass sie ihn, den Schlafenden, drinnen in seinem Bett erschlagen hätten. Aber deutsche Männer erzählen, sie hätten ihn draußen im Wald getötet. Und im alten Gudrunlied wird gesagt, dass Sigurd und Gjukis Söhne zum Thing geritten waren, als er erschlagen wurde. Aber das erzählen alle gleich, dass sie ihn um die Treue betrogen und ihn erschlugen, den Liegenden und Unbewaffneten.” Übersetzung: Krause 2001, S. 133, Kursivierung im Original).

In der Skáldskaparmál der Snorra Edda wird die Nibelungensage knapp zusammengefasst (Jónsson 1907, S. 174-186) und Snorri erwähnt (Jónsson 1907, S. 186):

„Eptir bessum sögum hafa flest skáld ort ok tekit ýmsa Páttu. Bragi hinn gamli orti um fall Sörla ok Hamðis í drápu Peiri, er hann orti um Ragnar loðbrók.”

(„Nach diesen Geschichten haben die meisten Skalden gedichtet und verschiedene Episoden daraus verwendet. Bragi der Alte besang den Fall Sörlis und Hamdirs, in dem Gedicht, das er über Ragnar lodbrok machte.” Übersetzung: Krause 1997, S. 153) 


\section{Die Prolegomena ad Homerum Friedrich August Wolfs}

Die Überlieferung des Nibelungenliedes endet mit dem so genannten ,Ambraser Heldenbuch“ (entstanden 1504-1515/16). Danach geriet das Epos für zweieinhalb Jahrhunderte in Vergessenheit, bis zunächst die Handschrift C 1755 in Hohenems wiederentdeckt und das Nibelungenlied durch Jakob Bodmer mit Homers ,Ilias“ in Verbindung gebracht wurde (grundlegend Pfalzgraf 2003). „In einer langen Reihe von Publikationen hat Bodmer die Wahrnehmung des ,Nibelungenliedes“ in der Perspektive der HomerForschung vorbereitet, die dann mit LACHMANNS Abhandlung von 1816 zum Ausgangspunkt des Germanistenstreits über die Bedeutung der Hss. werden sollte.“ (Heinzle 2014, S. 96)

Zwischen einer ersten Teilausgabe des Nibelungenliedes durch Bodmer 1757 (,Chriemhilden Rache, und die Klage') und der ersten neuzeitlichen Gesamtausgabe (1782 durch Christoph Heinrich Müller, vgl. Pfalzgraf 2003, S. 100-108) erschienen 1760-1763 die Gesänge Ossians, „die enthusiastisch als nordisch-autochthones Analogon zu Homer rezipiert wurden“ (Dehrmann 2019, S. 284), sich aber später als Fälschungen James Macphersons (1736-1796) und nicht als die postulierten gälischen Heldengesänge eines nordischen Homers aus dem dritten Jahrhundert herausstellten. Gleichwohl galt Ossian Herder als „Verkörperung des schöpferischen Genies schlechthin“ (Weber 2001, S. 163) und der Niederschlag der Ossian-Debatte auf die Herder'sche Theorie des Volksliedes (vgl. Gelbart 2011, S. 102-107) ist unverkennbar. Noch 1795 schreibt Herder von Ossian als dem „Homer des Nordens“ (Homer und Ossian, in: Die Horen).

Im gleichen Jahr erschienen Friedrich August Wolfs Prolegomena ad Homerum (Wolf 1795) und es ist aus heutiger Sicht kaum mehr zu erahnen, „welche Erschütterung jenes Ereignis für diese Zeit bedeuten mußte, das mit [Wolfs Werk] über die schöne Homerische Welt hereinbrach [...] Wolfs in nüchternem Latein systematisch aufgebaute These, die Ilias sei überhaupt nicht das Werk eines Mannes, sondern vieler, wirkte nun aber doch wie ein Denkmalsturz. Jeder war beeindruckt, doch in fast allen sträubte sich die dichterische Intuition gegen die verstandesmäßige Zerstückelung. Goethe etwa sprach zunächst von Wolf nur als von ,Homers Vernichter“. “(Latacz 2012, S. 112)

Nach Wohlleben (Wohlleben 1996, S. 155) fiel Wolfs Schrift „wie eine Bombe in eine friedliche Landschaft innigster deutscher Homerverehrung“. 
Den Grund fasst Heinzle (Heinzle 2014, S. 125) zusammen: „Das Homerische Epos, so lehrte Wolf, sei ein heterogenes Gebilde, eine sekundäre Verbindung traditioneller, ehedem mündlich tradierter Lieder verschiedener Verfasser, die einzelne Episoden des erzählten Geschehens behandelten. Das ergab sich für ihn zwingend aus Brüchen, Widersprüchen, Unebenheiten im Gefüge der Texte. Die Wirkung der Schrift war immens. Sie schreckte nicht nur die Philologen auf, sondern griff massiv in den poetologischen Diskurs der Zeit ein [...]“(vgl. auch Dehrmann 2019, S. 284)

Wolfs aufregende Kernaussage lautet also, dass sich in den überlieferten Texten nicht das „Dichtergenie“ eines Einzelnen, sondern die „vereinten Bemühungen vieler“ über einen langen Zeitraum hinweg manifestieren. Hierzu zählen im mündlichen Bereich Sänger (Aioiden), die episodische Einzellieder aus dem bekannten Sagenkreis gedichtet und vorgetragen hätten und die dann von anderen Sängern übernommen, variiert und überliefert wurden. Zur Zeit des Peisistratos (6. Jh. v. Chr.) haben der Theorie zufolge Rhapsoden Einzelgesänge der mündlichen Tradition vorgetragen, die nun schriftlich fixiert und durch Ordner (Diaskeuasten) zu größeren Zyklen angeordnet wurden. In weiteren Bearbeitungsschritten erfolgten Glättungen und weitere editorische Eingriffe. Zentral für die Wolf'sche Sichtweise ist der Ausgangspunkt, „dass das echte Epos nicht von einem einzelnen, als Individuum konturierten Autor stamme, sondern Produkt eines kollektiven Prozesses sei.“ (Dehrmann 2019, S. 285) In Auseinandersetzung mit der These Wolfs teilte sich die Homer-Forschung in zwei Lager: Auf der einen Seite standen die ,Analytiker' bzw. ,Traditionalisten', die Wolfs These vertraten, auf der anderen die ,Individualisten` bzw. Unitarier‘, die sie ablehnten (vgl. Heinzle 2014, S. 76; Haferland 2019, 37f.).

\section{Die Geburtsstunde der Germanistik: Zum Entstehungsprozess des Nibelungenliedes aus wissenschaftshistorischer Perspektive}

„Wenn, wie geschrieben steht, am Anfang das Wort, d.h. der Text war, folgte unmittelbar darauf Karl Lachmann (1793-1851), der, wenngleich klassischer Philologe von seiner Ausbildung, zusammen mit Jacob Grimm (1785-1863) den Bereich der deutschen Philologie (der einzigen „Germanistik“ zu dieser Zeit) gestaltete und definierte.“ (Gentry 2010, S. 605) Gentry spielt hier auf 
die Pionierarbeit im Bereich der Textkritik und die immense Editionsarbeit Lachmanns an, im germanistischen Bereich etwa die Iwein-Edition, die Edition der Gedichte Walthers von der Vogelweide, der Werke Wolframs von Eschenbach, Lessings - und des Nibelungenliedes (vgl. Münkler 2010).

Nach Lachmanns Dissertation 1814 in Halle - also dort, wo zwanzig Jahre zuvor die Prolegomena erschienenen waren - über Tibull und seiner Habilitation 1815 in Göttingen über Properz widmete er sich 1816 in seiner Berliner Probevorlesung der ursprünglichen Form des Nibelungenliedes die erste über ein mittelhochdeutsches Thema überhaupt. Im selben Jahr erschien Über die ursprüngliche Gestalt des Gedichts von der Nibelungen Noth, die den eigentlichen Beginn der deutschen Philologie markiert (vgl. Gentry 2010, S. 606). Darin übertrug Lachmann die oben skizzierte These Wolfs auf das Nibelungenlied: „Er war der Überzeugung, dieses sei keine einheitliche, in einem Guss entstandene Dichtung, sondern das Produkt eines Redaktors, der eine Reihe selbständiger Lieder von verschiedenen Verfassern, die jeweils einzelne Episoden des Geschehens behandelten, aneinanderfügte, ohne die Heterogenität des Materials restlos auflösen zu können.“(Heinzle 2014, S. 76) Lachmann formulierte seine Ansicht 1816:

„Ich glaube nämlich und werde in dem Folgenden zu beweisen suchen, daß unser so genanntes Nibelungenlied, oder bestimmter, die Gestalt desselben, in der wir es, aus dem Anfange des dreizehnten Jahrhunderts uns überliefert, lesen, aus einer noch jetzt erkennbaren Zusammensetzung einzelner romanzenartiger Lieder entstanden sei.” (Lachmann 1816, S. 3f.)

Die Übernahme der Wolf'schen These durch Lachmann führte zu einem langanhaltenden Richtungsstreit, dem sog. , Nibelungenstreit“ im 19. Jahrhundert, in dem es über das Nibelungenlied hinaus² letztlich „um das SelbstverständHaupthandschriften bzw. durch sie vertretenen Fassungen diskutiert (Heinzle 2014, S. 76). Die Handschriftenbezeichnungen bzw. Fassungen A, B, und C gehen auf Lachmann und deren von ihm vermuteten Verhältnis zum vorausgesetzten Original zurück. Vgl. als einführenden Überblick zu Lachmanns Textkritik Münkler 2010, S. 107-111. 
nis und die professionellen Standards der [Germanistik als] Disziplin“(Wyss 2001, S. 178) ging und der „bis in die gegenwärtige Forschung nach[wirkt]“. (Heinzle 2014, S. 76).

Erst zu Beginn des 20. Jahrhunderts erfolgte ein grundlegender Gegenentwurf zu Lachmann im Sinne eines echten Paradigmenwechsels: Andreas Heusler (1865-1940) entwickelte zunächst 1905 (Lied und Epos in germanischer Sagendichtung) eine Alternative, ,indem er an die Stelle der Textkritik die Sagengeschichte setzte“. (Wyss 2001, S. 179) Im Gegensatz zur Wolf-/ Lachmann‘schen (Klein-)Lieder- oder Sammeltheorie ging Heusler davon aus, dass das Epos nicht aus einer Vielzahl einzelner, sondern der Anschwellung eines Liedes hervorgehe:

„Der Weg vom Liede zum Epos ist Anschwellung; Verbreiterung des Stiles. Nach der Sammeltheorie verhält sich das Epos zum Liede wie eine Menschenreihe zum einzelnen Menschen; oder wie ein Baumspalier zum einzelnen Baume. Nehmen wir Epen und Lieder, so wie die Überlieferung der Germanen sie kennt, so müssen wir sagen: das Epos verhält sich zum Liede wie der erwachsene Mensch zum Embryo; wie der weitverästelte Baum zur jungen Pflanze.“ (Heusler 1905, S. 24)

Betrachtet man den idealtypischen Stammbaum Heuslers (Heusler 1955 [1921], S. 49), so zeigt sich eine bestechend einfache Entwicklung: Während Lachmann auf textkritischer Basis 1836 von 20 Einzelliedern ausging, sind es bei Heusler zwei Lieder, die durch allmähliche Angleichung schließlich vom Ependichter um 1200 im Buchepos verbunden wurden. In zwei (Brünhildlied) bzw. drei Stufen (Burgundenlied bis zur Älteren Nibelungennot) vom 5. bis ins 12. Jahrhundert zeichnet Heusler die Entwicklungsprozesse nach, die er durch Analyse der nordischen Zeugnisse gewinnt. Das Brünhildlied erreichte in Heuslers Modell beispielweise im 9. Jahrhundert den Norden und wird hier - ältere Sagenstufen werden in den Liedern der Edda verschiedentlich umgeformt und mit heimischem Erzählgut verwoben - variiert und akzentuiert. In der zweiten Stufe, die laut Heusler die Vorlage für das Nibelungenlied und die Thidrekssaga bildet, verlagert sich der Schwerpunkt auf Kriemhild (Gudrun). Die frühe Stufe des Burgundenliedes entspricht laut Heusler in 
den Grundzügen der Atlakvida, in der Zwischenstufe verschiebt sich das Rachemotiv von der Brüder- zur Gattenrache, Dietrich wird eingeführt und es entsteht nach Heusler um 1160 die berühmte Ältere Not, die den zweiten Teil des Nibelungenliedes ausmachte und über Soest auch Aufnahme in die Thidrekssaga erfuhr. Die von Heusler spekulativ eingeführte Ältere Not wurde - das zeigt die Wirkmächtigkeit Heuslers - „lange wie ein handschriftlich tradiertes Werk“(Schulze 2003, S. 70) behandelt.

Durch den im Norden angedeuteten, aber im Ganzen unterbliebenen und nur im Nibelungenlied erfolgten Verbinden zum Großepos wird ersichtlich, dass Heusler „den Ependichter als schöpferisches Individuum an die Stelle des bloß sammelnden und redigierenden Ordners“(Heinzle 2014, S. 78) setzt. Dadurch wird Heusler zum Wegbereiter der „individualistischen Forschungswende [...], die sich auch in einem terminologischen Wandel niederschlug: in der Ersetzung des alten Gattungsbegriffs ,Volksepos‘, dem der Gedanke einer überindividuellen Veranlassung der Texte zugrunde lag, durch den neuen, rein inhaltsbezogenen Begriff ,Heldenepos““. (Heinzle 2014, S. 78)

Die Stringenz von Heuslers Heldensagenmodell, das die lange Zeit vorherrschende Lachmann'sche Theorie ablöste, war derart bestechend, dass es in der ersten Hälfte des 20. Jahrhunderts quasi unangefochtene Lehrmeinung darstellte (vgl. Schulze 2003, S. 70). Unter anderem von Kuhn (Kuhn 1971) und Schröder (Schröder 1960) wurde es für seine Rigorosität kritisiert, und Walter Haug, der auf den „Denkzwang“ hinweist, der den Weg zu einem adäquaten Verständnis geradewegs verstelle (Haug 1989, S. 277), versuche seit „seit über zwanzig Jahren immer erneut, die heuslerianische Doxa zu überwinden“ (Wyss 2001, S. 174).

Zwanzig weitere Jahre später - in unserer Gegenwart - sind die Homerische und Heusler'sche Frage jeweils noch immer aktuell. Dies zeigt der 57-seitige Aufsatz von Harald Haferland mit dem Titel Das 'Nibelungenlied' im Zwischenbereich von Mündlichkeit und Schriftlichkeit, der 2019 in der Zeitschrift für deutsches Altertum erschien (Haferland 2019). Während sich Lachmann 1816 gleich im ersten Satz auf Wolf bezog, bezieht sich Haferland unmittelbar im ersten Satz auf Heusler: „Der Aufsatz richtet sich gegen das seit Andreas Heusler vorherrschende Verständnis des 'Nibelungenliedes’ als Buchepos und stellt den Begriff des Gedächtnistextes dagegen.” 
Harald Haferland greift ebenfalls auf den Vergleich von homerischen Epen und Nibelungenlied zurück: „'Ilias' und ,Nibelungenlied“ teilen das Geschick, dass mündliche Vorstufen in einen Text gelaufen sind, der schriftlich überkommen ist. Ungeklärt ist jeweils, wo, wie und in welchem Ausmaß die Verzahnung von Mündlichkeit und Schriftlichkeit stattgefunden hat. Unklar ist im Fall des ,Nibelungenliedes‘ auch, wie der mündliche Vorlauf überhaupt vorzustellen ist.“ (Haferland 2019, S. 79)

\section{Der finnische Homer oder: Lönnrots Kompilation. Von mündlicher Überlieferung zur Schriftkultur}

Im Falle des Nibelungenliedes lässt sich zusammenfassen, dass sich nach der langen mündlichen Überlieferung seit dem 5. Jahrhundert ab ca. 1200 die handschriftliche Überlieferung des Epos verfolgen und die in den Handschriften repräsentierten Fassungen bzw. Abhängigkeitsverhältnisse philologisch analysieren lässt.

Der Entstehungsprozess des Kalevala bietet eine - übrigens auch gerade dank Macpherson und der Ossian-Debatte (vgl. Haapoja-Mäkelä et al. 2018) - völlig andere Materialsituation. Neben der Nachverfolgbarkeit der ideengeschichtlichen Einflüsse aus Deutschland ist die politische Umbruchsituation in Finnland zu Beginn des 19. Jahrhunderts und das daraus resultierende nationale Programm bekannt - vor allem jedoch ist eine Analyse der mündlichen Dichtung (SKVR 1908-1948; 1997) und die minutiöse Dokumentation des Prozesses der Epenentstehung möglich. Heute stehen sowohl der Bestand der SKVR (Internetquelle [1]), als auch eine wissenschaftliche Edition des sogenannten Neuen Kalevala (Internetquelle [2]), ${ }^{3}$ als auch die Ausgabe des Briefwechsels Lönnrots (Internetquelle [3]) online über Portale der Finnischen Literaturgesellschaft zur Verfügung.

Als Elias Lönnrot 1822 sein Studium in Turku/Åbo aufnahm, hatten Henrik Gabriel Porthan und Christfrid Ganander bereits finnische Volkdichtung gesammelt und untersucht, der neuhumanistische Nachhall Porthans war weiterhin bedeutend (vgl. Nivala 2019). Wolfs Prolegomena gehörten zur Forschungstradition in Turku (vgl. Oksala 1990, S. 68) und die einflussreichen 
Schriften Herders hatten Turku direkt über Porthans Bibliothek erreicht (vgl. Kurkela 2012; Sommer 2012; Riikonen 2006). Von besonderer Bedeutung waren Herders Konzepte der Nation als Gemeinschaft, die von einer gemeinsamen Sprache individualisiert werde und ihren ,Charakter' in Sprache und Dichtung bewahre, der Geschichte als kontinuierlicher Bildungsprozess, weiter die Schätzung des Volksliedes und dessen Sammlung (vgl. Niedling 2021, S. 152).

Carl Axel Gottlund hatte 1817 in diesem Sinn angeregt, die mündlichen finnischen Gesänge systematisch zu sammeln und zu verbinden, damit „daraus ein neuer Homer, Ossian oder ein neues Nibelungenlied“ entstünde (zit. nach Schröder 2011, 245f., Übersetzung Schröder). Der Turkuer Historiker und Sprachforscher Reinhold v. Becker, dessen Schüler Lönnrot war, hatte 1820 den dreiteiligen Aufsatz „Väinämöisestä“ veröffentlicht, nachdem er Lieder über Väinämöinen gesammelt und zusammengefügt hatte. (vgl. Schoolfield 1998, S. 51).

In seiner Dissertation „De Väinämöine priscorum Fennorum numine“ (etwa: „Über Väinämöinen, eine Gottheit der alten Finnen“) aus dem Jahr 1827 beschrieb Lönnrot die gesamte bis dahin über den Helden des finnischen Altertums gesammelte Volksdichtung und begann, Verse aus verschiedenen Liedern ähnlichen Inhalts miteinander zu kombinieren. Durch den Stadtbrand in Turku im selben Jahr ging der zweite Teil dieser Arbeit verloren und durch den anschließenden Transfer der Universität nach Helsinki erfolgte eine akademische Zwangspause. Diese nutzte Lönnrot 1828 zur ersten von insgesamt elf Sammelreisen (vgl. Anttonen und Kuusi 1999, S. 25-29), die dem Zweck dienten, die finnische Volksdichtung zu dokumentieren. ${ }^{4}$ Zur systematischen Sammlung von Volksdichtung, der Förderung finnischer Kultur und des kulturellen Erbes wurde 1831 die Finnische Literaturgesellschaft mit Lönnrot als erstem Sekretär gegründet. So entstand der Rahmen für ein sehr ambitioniertes Projekt, das sich mit Lauri Honko als Kalevala-Prozess (vgl. Honko 2003, 2000b, Internetquelle [10]) beschreiben lässt.

4 In diesem Beitrag muss das hinter der Rückbesinnung auf mündliche Volksdichtung stehende nationale Programm zur besseren Fokussierung ausgelassen werden. Vgl. zu diesem Aspekt Saarelainen 2014; Haapoja-Mäkelä et al. 2018. 
Unter den vielen Sängern - von denen ein großer Teil nicht namentlich erwähnt wird - waren einige, die den jungen Lönnrot in besonderer Weise beindruckten und prägten. Senni Timonen (Timonen 2019, 2008) hebt u.a. Ontrei Malinen und Vaassila Kieleväinen von der vierten Sammelreise im September 1833 sowie Arhippa Perttunen hervor, den Lönnrot ein Jahr später traf. Ontrei, Vaassila und Arhippa - alle hatten den Sampo-Zyklus im Repertoire - spielen im Entstehungsprozess des Epos eine herausragende Rolle (vgl. Niedling 2020a, S. 463-467). Bei Ontrei aus Vuonninen ist der epische, durchkomponierte Vortrag des Sampo-Zyklus (SKVR I1: 79a) durch einen individuellen Stil geprägt, wozu ökotypisch die Verknüpfung des Sampo mit Pflügen und Säen und insbesondere die Wiederholung schöpferischer Akte am Ende des Zyklus gehören. Timonen beschreibt den Zyklus Ontreis im Umfang von 365 Versen als Kleinepos („,pienoiseepos“); (Timonen 2019).

Ganz anders war die Wirkung Vaassilas aus dem Nachbarort Vuokkiniemi. Lönnrot schreibt über sein Treffen mit ihm (Pentikäinen 1999, S. 100):
„Dieser Vaassila, hauptsächlich mit Zaubergesängen vertraut, war ein alter Mann. Sein Gedächtnis hatte in den letzten Jahren so nachgelassen, dass er nicht mehr so viel wusste wie früher. Über Väinämöinen und andere mythologische Figuren erzählte er jedoch viele Dinge, die mir vorher nicht bekannt waren. Und wenn es geschah, dass er etwas vergessen hatte, was ich bereits wusste, fragte ich ihn mehr dazu. Dann wiederum erinnerte er sich und so bekam ich das Wissen über alle Heldentaten Väinämöinens in einer Abfolge, und danach habe ich die Gesänge über Väinämöinen angeordnet, die bekannt sind.“

Der fragmentarische, unvollkommene, springende Charakter, der ganz verschiedene Sequenzen miteinander in Verbindung brachte (SKVR I1: 80) hat also nach Aussage Lönnrots die Anlage eines Plots gerechtfertigt bzw. mit den Worten Timonens: „Vaassila hatte gezeigt, dass die Gesänge kombinierbar waren, dass sich zwischen ihnen bedeutende zeitliche und 
inhaltliche Übereinstimmungen zeigten.“ ${ }^{5}$ Ca. 4 Monate später, im Dezember 1833, schrieb er an Henrik Cajander, dass er nun allein von Väinämöinen 5-6.000 Verse zusammenhabe, jedoch fortfahren wolle, bis er etwa „einen halben Homer“ (,en samling, som svarar emot 1/2 Homerus“; Internetquelle [4]) zusammen habe.

Wiederum nur wenige Wochen später, im Februar 1834, schrieb Lönnrot an den Vorsitzenden der Finnischen Literaturgesellschaft Henrik Gabriel Linsen über sein erwachtes Verlangen, die Gesänge zu einem „zusammenhängendem Ganzen zu ordnen“, aus der „finnischen Mythologie etwas der isländischen Edda entsprechendes zu bekommen [...] eine solche Sammlung, die unsere Nachkommen so hoch schätzen wie die gotischen Völker die Edda oder die Griechen und Römer, wenn nicht Homer, so zumindest Hesiod“ (Internetquelle [5]). ${ }^{6}$

Ein an Homer und anderen griechischen Epikern geschulter, mit Ossian und den Ideen Herders bekannter Akademiker war es also, der auf der fünften Sammelreise April 1834 in Latvajärvi Arhippa Perttunen traf. Von ihm sammelte er in drei Tagen 40 hervorragende Gesänge, darunter den längsten dokumentierten Sampo-Zyklus (SKVR I1: 54). Zu den bisherigen Eindrücken, die die Sänger durch ihre Persönlichkeit, den individuellen Stil und die Anordnung der Gesänge auf Lönnrot gemacht hatten, kam für Lönnrot in Kontakt mit Arhippa noch eine weitere Schlüsselerfahrung hinzu: der Bericht über die Sängerwettkämpfe des Vaters am Lapukka-See, deren Zeuge Arhippa als Kind wurde. ${ }^{7}$ Während der Abende und Nächte am Lagerfeuer maßen sich die erfahrenen Sänger mit ihrem Repertoire, ohne dass nach dem Bericht Arhippas je ein Gesang wiederholt worden wäre;

5 "Vaassila oli näyttänyt, että runot oli yhdistettävissä, että niiden väliltä löytyi merkitseviä ajallisia ja sisällöllisiä yhteyksiä.”

6 „[...] uppsteg hos mig begäret att ordna dem (efter) till ett helt sammanhängande för att derigenom uti Finska Mythologien få något motsvarande (Islandernas Edda) den Isländska Eddan [...] en sådan samling (framd) hos våra efterkommande möjligtvis kommer att värderas lika högt som hos de Göthiska folkslagen Eddan eller om hos Greker och Romare, om ej Homerus så åtminstone Hesiodus“.

7 Eine aufschlussreiche Darstellung dieser Passage inklusive einer Gegenüberstellung von Lönnrots direkten Feldnotizen mit den gedruckten Versionen bietet Saarinen 2018, S. 111-118; zum Phänomen der Sängerwettkämpfe vgl. auch Virtanen 1968, S. 14. 
der nach Lönnrots Bericht 80-Jährige ${ }^{8}$ saß als Junge am nächstgelegenen Lagerfeuer, lauschte und lernte.

Lönnrot verfügte nach dieser Reise über genug Material, eine epentheoretische Konzeption und einen Einblick in die Sängerwelt. 1835 erschien das so genannte Alte Kalevala in 32 Gesängen in einem Umfang von 12.078 Versen.

Was für ein historischer Zufall: "At the same time that Lachmann was deconstructing the German medieval epic ${ }^{9}$, Elias Lönnrot was assembling the Finnish epic he called Kalevala from shorter songs in conscious imitation of the Homer (or Pisistratus) described by Wolf.” (Haymes 2004, S. 43) Es ist jedoch wahrscheinlich, dass die Akteure in Berlin und Kajaani von der Arbeit des jeweils anderen keine Kenntnis hatten. Im Briefwechsel Lönnrots taucht Lachmann lediglich in einem Brief des ersten deutschen Übersetzers des Kalevala, Anton Schiefner, vom 29. März 1848 auf (Internetquelle [6]). Schiefner schildert darin, dass er zuerst aus den „Mittheilungen der esthnischen Gesellschaft zu Dorpat“ vom Kalevala erfuhr, unter Lachmann 1840-42 in Berlin die Nibelungen studierte und später über die Castrén'sche Übersetzung und insbesondere durch Jacob Grimms Akademie-Abhandlung Zugang zum Kalevala erhielt. Fromm wiederum hat gezeigt (vgl. Fromm 1990, S. 93-96), dass Lönnrot zwar früh solide Kenntnisse über die klassischen Sprachen erworben hatte, sein Deutsch aber rudimentär blieb und dass kaum Zweifel daran bestehen können, dass Lönnrot über Lachmanns WolfApplikation auf das Nibelungenlied nicht informiert war.

Zwischen 1835 und 1844 unternahm Lönnrot sechs weitere Sammelreisen und erhielt auch Material anderer Sammler wie Europaeus, Ahlqvist und anderen. 1845 beschäftigte sich Jacob Grimm in seiner berühmten Berliner Akademierede ausgiebig mit dem finnischen Epos. Durch die wiederentfachte Ossian-Debatte hatte die Finnische Literaturgesellschaft vor der Veröffentlichung des so genannten Neuen Kalevala, das mit 22.795 Versen in 50 Gesängen nahezu den doppelten Umfang des Alten Kalevala annahm, 1849 von Matthias Alexander Castrén (dem ersten Professor für finnische

8 Saarinen 2018 hat gezeigt, dass Arhippa 1834 deutlich jünger war - das vermeintlich höhere Alter mag den Eindruck auf Lönnrot noch einmal verstärkt haben.

$9 \quad$ Haymes bezieht sich auf Lachmann 1836. 
Sprache Literatur und damit Vorgänger Lönnrots auf dem Lehrstuhl) ein Gutachten bestellt, in dem dieser, wie später auch Europaeus, die direkten Aufzeichnungen der finnischen Gesänge ohne editorische Eingriffe empfahl (vgl. Anttonen 2015, S. 67).

Der ,finnische Homer legte erkennbar Wert darauf, nicht als ,finnischer Macpherson' zu gelten, obwohl er selbst-so wie schon Porthan-die Vorwürfe gegen Macpherson für unbegründet hielt (vgl. Anttonen 2015, Niedling 2021 und Honkos auf Lönnrot bezogenen Hinweis: "One may well ask: a fake of what?”; Honko 1990, S. 226 - vgl. dagegen die Vorstellung von einem singing scribe: Honko 2002, S. 18-20). Ein bekanntes Beispiel hierfür ist sein Brief an den französischen Übersetzer des Kalevala, Louis Antoine Léouzon Le Duc, vom 30. März 1851 (Internetquelle [7]):

„Ein solcher Zweifel zur Echtheit der Kalevalagesänge, wie er z. B. zu Kritik an der Echtheit von Macphersons ossianischen Gesängen führte, kann nicht bei jemandem entstehen, der wenigstens eine kleine Einsicht in die Bedingungen hat. Der Zweifel wäre dann auf die Sammler gerichtet, teils, dass sie den Originalgesang, den sie im Volk sammelten, veränderten und teils, dass sie die Herkunft der Gesänge verdeckten, die sie ordneten, oder ich könnte verdächtigt werden, dies anschließend getan zu haben, als ich das Kalevala formte. Dass dies nicht der Fall war, kann jedem, der dies wünscht bestätigt werden durch die Bevölkerung der Gegenden, wo die Gesänge gesammelt wurden, denn alles, was sich im Kalevala findet, war schon in einer oder einer anderen Region bekannt, und diese Bekanntheit ist gegründet auf Überlieferung und nicht durch irgendein gedrucktes oder geschriebenes Buch hervorgerufen, denn ein solches Buch existiert nicht in diesen Regionen, und es wäre den Finnen in den archangelischen und olonetzischen Gouvernements auch nutzlos, sie können nicht lesen und sind gleichwohl die wichtigsten Erhalter des Gesänge. Tatsächlich ist wahr, dass alles Material im Kalevala dem Bauern in der Region nicht einfach bekannt ist. Die Gesänge im Kalevala könnten noch immer gesammelt werden in der heutigen Generation, gäbe es jemanden, der mehrere Jahre mit Reisen und unter dem Volk verbringen wollte. 
Die zahlreichen Varianten desselben Gesanges von welchen einige bei der Finnischen Literaturgesellschaft sind, die meisten sich aber noch in meinem Besitz befinden mögen auch als Beweis für die Authentizität der Gesänge erwähnt werden, weil ein Fälscher sie sicherlich nicht zehn, zwanzig und oft sogar mehr Varianten der Gesänge produziert hätte. Während meiner editorischen Arbeit hatte ich einen solchen Variantenreichtum zur Verfügung, dass viele der Gesänge mit zwei oder mehr Paralleltexten von Anfang bis Ende hätten geliefert werden können, alle mit gleichem Inhalt aber so unterschiedlich in ihren Worten und Ausdrücken, dass sie abgesehen von den Namen wenig gemeinsam hätten.“10

\section{Lönnrots Ansicht war also: So lange die Verse des Kalevala in der Volksdichtung zu finden sind, sind Anspielungen auf Macpherson unbegründet. Die Varianten zeigen, dass von keiner als von einem Original gesprochen werden kann (vgl. Honko 1990, S. 225) und wohl auch, dass die Gesänge hinsichtlich}

10 Sådan misstanke om Kalevalarunornas äkthet, som t.ex. om äktheten af Macphersons ossianska sånger en tid sysselsatte kritici, kan ej gerna uppstå hos sågon, som helst litet känner förhållandet. Misstanken skulle då gälla dem, som uppsamlat runorna, att de dels förändrat de ursprungliga runor, som funnits hos folket, dels understuckit andra af dem sjelfva hopsatta, eller skulle jag misstänkas att sedermera vid ordnandet af dem till kalevala dikten hafva gjort sådant. Att så ej skett, kan dem som vill ännu öfvertyga sig om hos allmogen på de trakter, der runorna upptecknat, hvarest allt, som finnes i Kalevala, på den ena eller andra orten skall vara förut bekant, hvilken bekantskap åter är traditionel och ej härrörande af någon tryckt eller skrifven bok, då sådana ej finnas på samma orter, och dessutom skulle gagna till intet hos Finnarne i archangelska och olonetsiska guvernementet, hvilka ej kunna läsa, och hos hvilka dock runorna företrädesvis bibehållit sig. Ja det är så, att allting i Kalevala icke allenast är förut kändt hos allmogen på det nämda området, utan kunde Kalevala runorna ännu väl under den närvarande generationen ånyo uppsamlas, om någon under en flerårig vandring och vistelse hos folket hade lust dertill. Äfven de mångfalldiga varianterna af samma runor, som upptecknats, och finnas till någon del hos litteratursällskapet i Helsingfors men hufvudsakligast ännu hos mig, kunna anföras till intyg på runornas akthet, ty en förfalskare hade väl ej brytt sig om att hopdikta 10, 20 och ofta ännu flere sådana varianter. Under redaktionen fann jag en så rik tillgång af varianter, att mången runo nästan från början till slutet kunnat utrustas med tvänne eller flere parallera texter, hvilka väl till innehållet varit lika, men hvad orden och uttrycken beträffar, så olika lysande, att knappt annat än namnen varit gemensamma. 
der Erzählstruktur/ des Erzählgerüsts Ähnlichkeiten aufweisen. Honko weist darauf hin, dass Lönnrot sich berechtigt fühlte, dieselben Regeln wie die Sänger beim Gesang anzuwenden. Diese Regeln betrafen Variation und Handlungsgestaltung (vgl. Honko 1990, S. 222).

Anhand des Sampo-Zyklus wurde gezeigt (Niedling 2020b), dass Sänger Sequenzen und Lieder um und in einen relativ festen Plot aus einem gemeinsamen Sequenzbestand ein-bzw. anfügen, verketten und anschwellen können. Für das weißkarelische Kerngebiet des Sampo-Zyklus ergab sich z. B. bei den Familientraditionen Malinen und Perttunen in einem Zeitraum von 90 Jahren folgendes verfestigtes Schema (vgl. hierzu Niedling 2020b, S. 166):

NV $-(. .)-.F L-(\ldots)-(.)-.S S-R S$

Diese Sequenzen bilden die Struktur des Zyklus ab, die über Jahrhunderte eine verfestigte Überlieferungstradition bildete. Väinämöinen wird niedergeschossen und treibt in das Nordland, von wo er aus eigener Kraft nicht mehr zurückkehren kann. Er ist auf die Hilfe der Herrin des Nordlands angewiesen, die als Gegenleistung das Schmieden des Sampo fordert. Nachdem dieser fertig gestellt ist und Wohlstand erzeugt, wird er von Väinämöinen und Gefährten geraubt und geht im Abwehrkampf verloren bzw. wird zerstört.

In der Familientradition der Malinens hat sich eine auffällige Verbindung des Sampo mit dem Ritus von Pflügen und Aussaat etabliert. Iivana Malinen, Ontrei Malinens Enkel, berichtete dem Sammler, dass jeweils vor der Frühjahrs- und Herbstaussaat die Worte des Pflügens und anschließend die Gesänge vom Schmieden und Raub des Sampo gesungen wurden, gefolgt von dem expliziten Rückzug der Herrin des Nordlands und wie Väinämöinen den von ihr geschickten Frost unschädlich machte (vgl. Niedling 2020b, S. 91). Bei den Perttunens gibt es dagegen Hinweise auf die Konzeption eines neuen Sampo (vgl. Niedling 2020b, S. 147 und 166).

Für alle ausgewerteten Varianten Weißkareliens zeigen sich neben einem breiteren Spektrum möglicher Sequenzen auch in der Anordnung Variationsmöglichkeiten, die erzählstrategisch mit einer Verkettung zusammenhängen können:Vor dem üblichen Beginn bzw. der Eingangssequenz, dem Niederschießen Väinämöinens, kann der Sängerwettkampf mit Joukahainen stehen und nach dem üblichen Ende des Handlungsschemas, dem 
Raub des Sampo, können weitere Sequenzen folgen. Die Klammern zeigen, dass keine der Sequenzen immer realisiert ist und mögliche Leerstellen, die durch Sequenzen besetzt werden können (Niedling 2020b, S. 170):

\section{$(\ldots)-(\mathrm{NV})-($ FL u./od. EW $)-(\ldots)-(\mathrm{SS})-(\ldots)-(\ldots)-(\ldots)-(\ldots)-(\mathrm{RS})-(\ldots)-(\ldots)$}

Wie in dem oben dargestellten relativ verfestigten Beispiel der Malinens und Perttunens ist die Anordnung der Sequenzen und damit des SampoZyklus annähernd gleichbleibend, aber aus dem pool of tradition können mehr (optionale) Sequenzen (innerhalb des Erzählgerüstes oder an seinen Rändern) aktiviert und in den Zyklus integriert werden.

Lönnrot unterscheidet sich von den einzelnen Sängern der jeweiligen Regionen dadurch, dass sein überregional gesammeltes Repertoire sehr viel größer ist: „Die traditionellen Sänger (rune singers) kombinierten in der Tat Gesänge, um eine logische Abfolge $\mathrm{zu}$ formen und dabei gab es bemerkenswerte Übernahmen von Episoden aus verschiedenen Gesängen, aber Lönnrot gab dieser Möglichkeit völlig neue Dimensionen (Honko 2000a, S. 223). Vielleicht können wir Lönnrot hier als Primus inter pares (vgl. Niedling 2020a) oder wie Honko als singing scribe (Honko 2002, S. 18-20) auffassen.

Der Ossian-Debatte ist es also zusammenfassend zu verdanken, dass der fünfstufige Kalevala-Prozess Elias Lönnrots bestens dokumentiert wurde (vgl. Apo 2004, 273f.; 276). Lönnrot erlaubte und wollte es, dass wir ihm bis in jede Einzelheit über die Schulter schauen können. Aus diesem Grund kann der Kompilationsprozess auch in Zahlen ausgedrückt werden: Ca. 33 \% der Verse des (Neuen) Kalevala sind identisch mit der gesammelten Volksdichtung, 50 \% wurden von Lönnrot sprachlich, orthographisch oder metrisch angepasst, 14 \% sind Zusammenstellungen Lönnrots aus der Volksdichtung, 3 \% sind Eigendichtung Lönnrots (vgl. Järvinen 2017, S. 73).

\section{Der mentale Text: traditionsorientierte Epen im Zwischenbereich von Mündlichkeit und Schriftlichkeit}

Im Falle der karelischen Sänger wurde die Bedeutung und individuelle Eigenleistung Ontreis, Vaassilas und Arhippas hervorgehoben. Sie trugen ihre Varianten des Sampo-Zyklus vor. Etwas sehr ähnliches formulierte 
Andreas Heusler für die Nibelungen-Überlieferung (Heusler 1955 [1921], S. 111, Sperrung im Original):

„Eben darum ist der sachlich richtige Ausdruck vom Heldensänger der alte, volkstümliche: 'er singt das L i e d von Kriemhildens Verrat' usw. Das, nicht ein! Dieses Lied klang um 800 sehr anders als um 1200, es klang in Passau anders als in Soest, anders im Munde des Kuonrat als in dem des Eberhart - und war doch eine Einheit, ein Lebewesen wie die Raupe in ihren fünf Häutungen.”

Heusler umreißt die Überlieferungsgebundenheit des Stoffes, hier das Heldenlied, das seine Identität behält und dabei im Laufe der Zeit bei regionalen Schwerpunktbildungen durch das Sänger-Individuum umgeformt wird. Motive verschieben, überlagern oder verlieren sich, Figuren gewinnen an Bedeutung oder verblassen, Sagen finden durch innere und äußere Anbindung zueinander.

Der Sänger greift im mündlichen Akt seiner individuellen Performanz auf einen Vorrat bestehender Phrasen, Formeln und Motive, einen Rahmen, Bilder, Multiformen - ein gemeinsames Register zurück. Lauri Honko schlägt hierfür den Begriff des mentalen Textes vor. Seine Definition lautet (Honko 1998, S. 94):

„To be able to understand the production of text in actual performance, it seems necessary to postulate a kind of "prenarrative", a pretextual frame, i.e., an organised structure of relevant conscious and unconscious material present in the singer's mind. This preexistent module seems to consist of 1) storylines, 2) textual elements, i.e., episodic patterns, images of epic situations, multiforms, etc., and 3) their genetic rules of reproduction as well as 4) contextual frames such as remembrances of earlier performances, yet not as haphazard collection of traditional knowledge but, in the case of distinct epics of the active repertoir, a prearranged set of elements internalised by the individual singer. We may call this variable template a "mental text", an emergent entity, able to be cut to different sizes and adapted to various modes of performance yet preserving its textual identity.” 
Honko ergänzt (a.a.O., S. 95), dass auch der einzelne Hörer im Publikum über einen eigenen mentalen Text, mentale Bilder und persönliche Interessen und Vorlieben verfügt (vgl. auch oben Schmid 2018). Zu einem sehr ähnlichen Bild kommt Haferland, wenn er „die Kopfbühnen des Sängers und der Zuhörer“ bzw. die „Imaginationsbühne“ der Zuhörer und die „Gedächtnisbühne“ des Sängers (Haferland 2019, S. 68) beschreibt, die Sequenzen überschreitend eine Rahmenbildung erlauben. Das Nibelungenlied ist für Haferland weniger ein Buchepos nach Heusler'schem Verständnis als ein „aufgezeichneter Gedächtnistext“ (Haferland 2019, S. 29).

Foley (1999, Internetquelle [8]) hebt bei Honkos Konzept des mentalen Textes hervor, dass es die Perspektive von der kollektiven Überlieferung der etablierten Sänger-Instanz hin zu dem einzelnen vortragenden SängerIndividuum verschiebe (,[Honko] he starts by shifting the perspective from the collective tradition, as instanced in song- performances by a variety of bards, to the individual performer").

Hakamies (Hakamies 2014, 16f.) merkte an, dass Honko kein Interesse zeigte, sein Konzept mit kognitiven Theorien in Verbindung zu bringen, was jedoch später von Palmieri (vgl. Palmieri 2017, insbesondere 197-201) durch Übertragung auf deklarative und prozedurale Prozesse durchgeführt wurde. Kritik gegen Honkos Konzept des mentalen Textes wurde auch hinsichtlich terminologischer Aspekte (Foley 1999) und seines vorgeblich eingeschränkten Wertes bei der Gewichtung der Textquellen Lönnrots (vgl. Apo 2004, S. 286-291) angeführt.

Die für Heusler beschriebene Bedeutung des schöpferischen Individuums bei der Ependichtung hebt auch Lauri Honko hervor - im mündlichen wie im semi-oralen Bereich (vgl. Honko 1998, S. 34, Internetquelle [9]). Honko modifizierte die Oral Formulaic Theory mit einer Ausrichtung auf semi-orale Epik (vgl. Foley 2004). Mit Bezug auf Lönnrot bedeutet das konkret, dass das auf mündlicher Überlieferung basierende Kalevala Elias Lönnrots nicht ein Buch (oder fünf Bücher), sondern in bestimmter Hinsicht kein Buch sei (Honko 2002, S. 15): „It is a peculiar mental text in Elias Lönnrot's mind, developed in interaction with the epic registers of different regions as manifested by singers whom he met or recieved materials from." Diese Sichtweise führt zum Sänger-Individuum traditionsorientierter Epen. Dabei bleibt letztlich auch für das Kalevala zu berücksichtigen (Heinzle 2014, S. 126, Sperrung im 
Original): „Der Streit [um Homer als Originalgenie in Auseinandersetzung zu Wolfs These] ist bis heute nicht entschieden - er kann gar nicht entschieden werden, weil die Alternative falsch ist. Wer die Epen in ihrer historischen Eigenart verstehen will, muss sie aus b e i d e $\mathrm{n}$ Perspektiven in den Blick nehmen: aus der traditionalistischen u $\mathrm{n}$ d aus der individualistischen. Jede Beschränkung auf die eine oder die andere führt zu Fehlurteilen, verhindert Erkenntnis. Wenn die Forschungsgeschichte der Homerischen Frage eines lehrt, dann dies. Die Mediävisten sind gut beraten, sie zu studieren, wenn sie wissen wollen, was sie tun und was sie zu tun haben, wenn sie sich mit Werken des Genres befassen, mit der 'Chanson de Roland' z. B. oder eben dem ,Nibelungenlied'“.

\section{Zusammenfassung}

In Auseinandersetzung mit den Prolegomena von Friedrich August Wolf wurde in diesem Beitrag an den Beispielen Nibelungenlied und Kalevala die Frage verfolgt, welchen Entstehungsbedingungen traditionsorientierte Epen unterliegen und welche Entstehungsmodelle z. T. bis heute den Forschungsdiskurs beeinflussen. Im Falle des Nibelungenliedes bedeutete dies, dass man seit dem 19. Jahrhundert seine Genese zu rekonstruieren versuchte, während das Kalevala ein Produkt Wolf'scher und Herder'scher Einflüsse darstellt, in dem Elias Lönnrot finno-karelische epische Dichtung zum Epos kompilierte. Die Dichtung der Sänger hinter dem Kalevala, die man als Miniatur- oder Mikro-Epen auffassen kann (vgl. Timonen 2019; Hatto 1991), ist aufgrund der gemeinsamen Gattungszugehörigkeit mit dem Nibelungenlied geeignet, einander entgegen stehende Entstehungstheorien wie die von Lachmann und Heusler anzunähern (vgl. Niedling 2020b, S. 183-193). Die finno-karelische Materialsituation erlaubt ferner den Fokus auf den Sänger und seinen ganz individuellen, Varianz hervorrufenden Umgang mit der kollektiven Überlieferung zu richten. Seine Interpretation der Überlieferung kann mit Honko als mentaler Text beschrieben werden.

Elias Lönnrot, der Kompilator des Kalevala, nimmt hier eine besondere Position ein. Auf der einen Seite waren ihm Wolfs Thesen und epische Vorbilder vertraut, benutzte er das Medium der Schrift, ja: war er auf dieses 
für den eigenen Epenprozess angewiesen. Auf der anderen Seite ist er als singing scribe selbst Sänger mit unerreichter Einsicht in die Sängerwelt.

Verbindet man den mentalen Text enger mit der kognitiven Theorie, ergeben sich Annäherungen zu Haferlands Betrachtung des Nibelungenliedes als Gedächtnistext. Das Spektrum denkbarer Möglichkeiten im Verschriftungsoder Verschriftlichungsprozess ist breit: „Zwischen einer nachträglichen oder zum Vortrag parallelen Aufzeichnung (Verschriftung) solcher Texte [mündliche Volkserzählungen], die ohne Zwischenschaltung von Schrift entstanden sind, und einem über ein Schriftmedium in Anlehnung an ein Schreibformat oder eine Gattung mehr oder weniger Wort für Wort geplanten Text erstreckt sich ein weitläufiges Kontinuum abgestufter Möglichkeiten.“ (Haferland 2019, S. 80) Der Entstehungsprozess des Kalevala ist zweifellos als Sonderform zu behandeln, erlaubt jedoch trotz dieser Einschränkung einen wertvollen Blick auf das Sänger-Individuum im mündlichen wie im semi-oralen Bereich. $\mathbf{N}$

CHRISTIAN NIEDLING

UNIVERSITÄT HELSINKI 


\section{Literaturverzeichnis}

\section{Internetquellen (alle zuletzt aufgerufen am 29.04.2021)}

[1]: https://skvr.fi/.

[2]: http://kalevala.finlit.fi/.

[3]: http://lonnrot.finlit.fi/omeka/.

[4]: http://lonnrot.finlit.fi/omeka/ items/show/2287.

[5]: http://lonnrot.finlit.fi/omeka/ items/show/1139.

[6]: http://lonnrot.finlit.fi/omeka/ items/show/7324.

[7]: http://lonnrot.finlit.fi/omeka/ items/show/7805.

[8]: https://www.folklorefellows.fi/ experiencing-the-siri-epic (1999).

[9]: https://www.folklorefellows.fi/the-fiveperformances-of-the-kalevala/ (2000).

[10]: https://matkallakalevalaan.finlit.fi/ kalevala-monta/viisi-kalevalaa (2020).

[11]: https://kaku.kalevalaseura.fi/lonnrotja-runonlaulun-estetiikka/ (2019).

AntTonen, Pertti 2015. The Kalevala and the Authenticity Debate. Manufacturing a past for the present. Forgery and authenticity in medievalist texts and objects in nineteenth-century Europe, Hrsg. János M. Bak/Patrick J. Geary/ Gábor Klaniczay. Leiden/Boston. 56-80. https://doi.org/ 10.1163/9789004276819_004

AnTtOnEn, Pertti/Kuusi, Matti (Hrsg.) 1999. Kalevala-Lipas. Jyväskylä. APO, Satu 2004. Laulaen vai kirjallisesti luoden? Uuden Kalevalan valmistusprossessi Elias Lönrotin kuvaamana. Kalevala ja laulettu runo, Hrsg. AnnaLeena Siikala/Lauri Harvilahti/Senni Timonen. Helsinki. 273-298.
DEHRMANN, Mark-Georg 2019: Galerie der Volksgeister. Zum europäischen Diskurs des ,Nationalepos‘ im 19. Jahrhundert. Zeitschrift für Germanistik (2): 282-303. https://doi.org/10.3726/92165_282 FOLEY, John Miles 2004. Epic as genre. In: R. L. Fowler (Hg.): The Cambridge companion to Homer, Hrsg. Robert Fowler. Cambridge. 171-187. https:// doi.org/10.1017/CCOL0521813026.011 FROMM, Hans 1990. Kalevala and Nibelungenlied: The Problem of Oral and Written Composition. Religion, Myth, and Folklore in the World's Epics. The Kalevala and its Predecessors, Hrsg. Lauri Honko. Berlin/ New York. 93-114. https:// doi.org/10.1515/9783110874556.93

GELBART, Matthew 2011. The invention of "folk music" and "art music". Emerging categories from Ossian to Wagner. Cambridge/New York/Melbourne. GENTRY, Francis 2010. German Studies. Handbook of medieval studies. Terms, methods, trends, Hrsg. Albrecht Classen. Berlin. 602-618. https:// doi.org/10.1515/9783110215588.602 HAAPOJA-MÄKELÄ, Heidi/Stepanova, Eila/ Tarkka, Lotte 2018. The Kalevala's Languages: Receptions, Myths, and Ideologies. Journal of Finnish studies (21): 15-45.

HAFERLAND, Harald 2019. Das

'Nibelungenlied' im Zwischenbereich von Mündlichkeit und Schriftlichkeit. ZfDA (148): 28-84. https://doi.org/ 10.3813/zfda-2019-0003

HAKAMIES, Pekka 2014. Innovations in Epic Studies by Lauri Honko. Approaching Religion 4 (1): 13-17. https://doi.org/10.30664/ar.67532 
HATTO, Arthur 1991. Eine allgemeine Theorie der Heldenepik. RheinischWestfälische Akademie der Wissenschaften, Vorträge, G307. 7-24. https:// doi.org/10.1007/978-3-663-01808-7_1

HAUG, Walter 1989 [1975]. Andreas

Heuslers Heldensagenmodell: Prämissen, Kritik und Gegenentwurf. Strukturen als Schlüssel zur Welt. Kleine Schriften zur Erzählliteratur des Mittelalters. Hg. v. Walter Haug. Tübingen, S. 277-292.

HAYMES, Edward R. 2004. The Germanic Heldenlied and the Poetic Edda: Speculations on Preliterary History. Oral Tradition (19/1): 43-62. https://doi.org/10.1353/ort.2004.0091 HEINZLE, Joachim 2005. Wiedererzählen in der Heldendichtung: zur Fassung n des ,Nibelungenliedes' ZfdPh 124: 139-158.

HEINZLE, Joachim (Hrsg.) 2014.

Traditionelles Erzählen. Beiträge zum Verständnis von Nibelungensage und Nibelungenlied (= Zeitschrift für deutsches Altertum und deutsche Literatur, Beiheft 20). Stuttgart. HEUSLER, Andreas 1905. Lied und Epos in germanischer Sagendichtung. Dortmund.

HEUSLER, Andreas 1955 [1921]. Nibelungensage und Nibelungenlied. Die Stoffgeschichte des deutschen Heldenepos. 5. Aufl. Dortmund. HONKO, Lauri 1990. The Kalevala: The Processual View. Religion, Myth, and Folklore in the World's Epics. The Kalevala and its Predecessors, Hrsg. Lauri Honko. Berlin/New York. 181-230. https://doi.org/ 10.1515/9783110874556.181

HONKO, Lauri 1995. Problems of oral and semi-literary epics. Formen und Funktion mündlicher Tradition. Vorträge eines Akademiesymposiums in Bonn, Juli 1993, Hrsg. Walther Heissig. Opladen. 26-40. https://doi.org/ 10.1007/978-3-322-84033-2_2
HONKO, Lauri 1998. Textualising the Siri Epic. Helsinki.

HONKO, Lauri 2000a. Text and context in the textualization of Tulu oral epics. Textualization of oral epics, Hrsg. Lauri Honko. Berlin. 217-236. https://doi.org/10.1515/9783110825848

HONKO, Lauri 2000b. The five performances of the Kalevala. [= Internetquelle 9].

HONKO, Lauri 2002. The Kalevala as performance. The Kalevala and the world's traditional epics, Hrsg. Lauri Honko. Helsinki. 13-25.

HONKO, Lauri 2003. The Quest for the Long Epic. Three Cases. Dynamics of Tradition. Perspectives on Oral Poetry and Folk Belief, Hrsg. Lotte Tarkka. Helsinki. 191-212.

HONKO, Lauri/Timonen, Senni/Branch, Michael (Hrsg.) 1993. The Great Bear. A Thematic Anthology of Oral Poetry in the Finno-Ugrian Languages. Helsinki. JóNSSON, Finnur (Hrsg.) 1907. Edda Snorra Sturlusonar. Reykjavík.

JÄRVINEN, Irma-Riitta 2017. Kalevala guide. 2. Aufl. Helsinki.

KRAUSE, Arnulf (Hrsg.) 1997. Die Edda des Snorri Sturluson. Stuttgart.

KRAUSE, Arnulf (Hrsg.) 2001. Die Heldenlieder der Älteren Edda. Stuttgart.

KUHN, Hans 1971 [1952]. Heldensage vor und ausserhalb der Dichtung. Hans Kuhn: Kleine Schriften. Aufsätze und Rezensionen aus den Gebieten der germanischen und nordischen Sprach-, Literatur- und Kulturgeschichte. Zweiter Band. Berlin (2), S. 102-118. KURKELA, Vesa 2012. Sorrowful Folksong and Nationalism in NineteenthCentury. Folklore and nationalism in Europe during the long nineteenth century, Hrsg. Timothy Baycroft/ David M. Hopkin. Leiden/Boston. 351-370. https://doi.org/ $\underline{10.1163 / 9789004211834 \_018}$ 
LACHMANN, Karl 1836. Zu den Nibelungen und zur Klage. Berlin. https://doi.org/ $10.1515 / 9783111496177$

LATACZ, Joachim 2012. Homer und Europa. Höhepunkte Homerischen Einflusses auf Europas Kulturentwicklung. Homer, gedeutet durch ein grosses Lexikon. Akten des Hamburger Kolloquiums vom 6.-8. Oktober 2010 zum Abschluss des Lexikons des frühgriechischen Epos, Hrsg. Michael Meier-Brügger. Berlin. 89-118. https://doi.org/ $\underline{10.1515 / 9783110292572.89}$

MÜNKLER, Marina 2010. „durch unverdrossene tüchtige Arbeit": Karl Lachmann (1793-1851) als Philologe. Zeitschrift für Germanistik 20/1: 104122. https://doi.org/10.3726/92128_104 NECKEL, Gustav (Hrsg.) 1914. Edda. Die Lieder des Codex Regius nebst verwandten Denkmälern. Heidelberg. NIEDLING, Christian 2020a. Von Halle nach Finnland oder: Wenn man anhand deutscher Epentheorien den karelischen Sänger belauscht. „In vriuntschaft als es was gedâht“. Freundschaftsschrift für Hans-Joachim Solms, Hrsg. Ammer, Jessica/Meiser, Gerhard/Link, Heike. Berlin. 454-472. NIEDLING, Christian 2020b. Epentheorie als interdisziplinäre Methodologie. Die Epentheorien Karl Lachmann und Andreas Heuslers vor dem Hintergrund karelischer Sampo-Epik und eddischer Überlieferungen. Dissertation. Åbo.

NIEDLING, Christian 2021. Eine versteckte Philologie. Zur Entwicklung der „nationalen Wissenschaften“ Finnlands. Zeitschrift für deutsche Philologie (Sonderheft 139: Deutsche Philologie? Nationalphilologien heute): 147-163. NIVALA, Asko 2019. Eurooppalainen romantiikka ja Kalevala. Eurooppa, Suomi, Kalevala. Mikä mahdollisti
Kalevalan?, Hrsg. Ulla Piela/Pekka

Hakamies/Pekka Hako. Helsinki. 15-37. OKSALA, Teivas 1990. Virgil's Aeneid as Homeric, National and Universal Epic. Religion, Myth, and Folklore in the World's Epics. The Kalevala and its Predecessors, Hrsg. Lauri Honko. Berlin/ New York. 49-72. https://doi.org/ $\underline{10.1515 / 9783110874556.49}$

PALMIERI, Giacinto 2017. Oral selftranslation of stand-up comedy and its (mental) text: a theoretical model. HUMOR 30: 193-210. https://doi.org/ 10.1515/humor-2016-0092

PENTIKÄINEN, Juha 1999. Kalevala Mythology. Expanded Edition. Bloomington/Indianapolis. PFALZGRAF, Annegret 2003. Eine Deutsche Ilias? Homer und das „Nibelungenlied" bei Johann Jakob Bodmer. Zu den Anfängen der nationalen Nibelungenrezeption im 18. Jahrhundert. Dissertation. Marburg. Tectum. RIIKONEN, Hannu 2006. J. G. Herderin tuntemus Turun Akatemian piirissä Porthanin ja Franzénin aikana. Herder, Suomi, Eurooppa, Hrsg. Kari Immonen/ Sakari Ollitervo. Helsinki. 265-290.

SAARELAINEN, Juhana 2014. Becoming of a Memory Box: the Kalevala. Sung Poetry, Printed Word and National Identity. Memory boxes. An experimental approach to cultural transfer in history, 1500-2000, Hrsg. Heta Aali/Anna-Leena Perämäki/ Cathleen Sarti/Jörg Rogge/Hannu Salmi. Bielefeld. 155-175. https://doi.org/10.14361/ transcript.9783839427866.155

SAARINEN, Jukka 2018. Runolaulun poetiikka. Säe, syntaksi ja parallelismi Arhiippa Perttusen Runoissa. Dissertation. Helsinki. https://doi.org/10.30666/elore.72825 scHMID, Florian 2018. Die Fassung ${ }^{*} \mathrm{C}$ des $>$ Nibelungenlieds $<$ und der $>$ Klage $<$. 
Strategien der Retextualisierung.

Berlin. https://doi.org/

$10.1515 / 9783110593020$

SCHOOLFIELD, George C. 1998. A history of

Finland's literature. Lincoln/ London.

SCHRÖDER, Franz Rolf 1960. Siegfrieds

Tod. GRM 41: 111-122.

SCHRÖDER, Stephan Michael 2011.

1809 aus deutscher Perspektive:

Rühs' Finland und seine Bewohner.

1809 und die Folgen. Finnland

zwischen Schweden, Russland und

Deutschland, Hrsg. Jan Hecker-

Stampehl/Berns Henningsen/Anna-

Maija Merten/Stephan Michael

Schröder. Berlin. 229-248.

SCHULZE, Ursula 2003. Das Nibelungenlied.

Durchgesehene und bibliographisch

ergänzte Ausgabe. Stuttgart.

SKVR. Suomen kansan vanhat runot

1908-1948; 1997. 15 Bände. Helsinki.

SOMMER, Łukasz 2012. A Step Away from

Herder: Turku Romantics and the

Question of National Language. The

Slavonic and East European Review

(90/1): 1-32. https://doi.org/

10.5699/slaveasteurorev2.90.1.0001

TIMONEN, Senni 2008. Elias Lönnrot ja

runonlaulaja. Kalevalan

kulttuurihistoria, Hrsg. Ulla Piela/

Seppo Knuutila/Pekka Laaksonen.

Helsinki. 2-27.

TIMONEN, Senni 2019. Lönnrot ja runonlaulun estetiikka (aktualisierte Online-Version von Timonen, Senni 2008 = Internetquelle [11]).

WEBER, Gerd Wolfgang 2001. Mythos und

Geschichte. Essays zur Geschichts-

mythologie Skandinaviens in

Mittelalter und Neuzeit. Triest.

VIRTANEN, Leea 1968. Kalevalainen

laulutapa Karjalassa. Helsinki.

WOHLLEBEN, Joachim 1996. Friedrich

August Wolfs Prolegomena ad

Homerum in der literarischen Szene der Zeit. Poetica (28): 154-170. https:// doi.org/10.30965/25890530-0280102006 WOLF, Friedrich August 1795.

Prolegomena ad Homerum sive de operum Homericorum prisca et genuina forma variisque mutationibus et probabili ratione emendandi. Halle.

WYSS, Ulrich 2001. Nibelungische

Irritationen. Das Heldenepos in der Literaturgeschichte. 6. Pöchlarner Heldenliedgespräch. 800 Jahre Nibelungenlied: Rückblick, Einblick, Ausblick, Hrsg Klaus Zatloukal. Wien. 171-186. 